\title{
Monitoring Autonomic Progress Using Outlier Analysis of Heart Rate Variability: A Case Study
}

\author{
John Hart* \\ South Carolina, USA
}

*Corresponding author: John Hart, Hart Chiropractic, Greenville, South Carolina, USA

\section{Abstract}

This report describes a method of monitoring neurological (autonomic) progress (or lack thereof) where two different natural interventions were used for an individual patient: Chiropractic care and exercise. Not much progress was made initially with the chiropractic care but was observed after the implementation of exercise, evidenced in this case by outlier analysis. Though this progress was short-lived, it nonetheless suggests that the intervention of exercise should be more fully explored

\section{Introduction}

Heart rate variability (HRV) is a measure of autonomic health, where in general, greater variability indicates a healthier (more adaptive) autonomic nervous system [1]. Natural methods of improving HRV include chiropractic care [2-3] and exercise [4]. This case study pertains to a patient of the author's who initially received chiropractic care and then initiated an exercise program. The purpose of the study was to: a) demonstrate how HRV can be used on a visit-by-visit basis to monitor neurological progress (or lack thereof) following different interventions, and b) show how outlier analysis can be used to determine the statistical unusualness of an apparent substantive change in HRV compared to baseline.

\section{Case Presentation}

A 50-year-old adult female consulted the author in the April of 2019 complaining of low back pain. The author explained that his practice objective was to determine if she had a chiropractic subluxation, conceptually defined as a slight vertebral misalignment that is disturbing nearby spinal nerves and/or cord. If a subluxation is found, then a chiropractic adjustment is recommended to improve neurological function so patients can better heal themselves of what ails them. The patient consented to care and to this case report. The operational definition of subluxation in the study was based on the exam procedures at Hart Chiropractic which included: a) neurological (autonomic) assessment using HRV and b) spinal alignment assessment using manual palpation. The neurological assessments were used for determining when to adjust while the palpation was used for direction of the misalignment. The author's focus is on the upper cervical spine, specifically the atlas (C1) so that is where the palpation was applied.

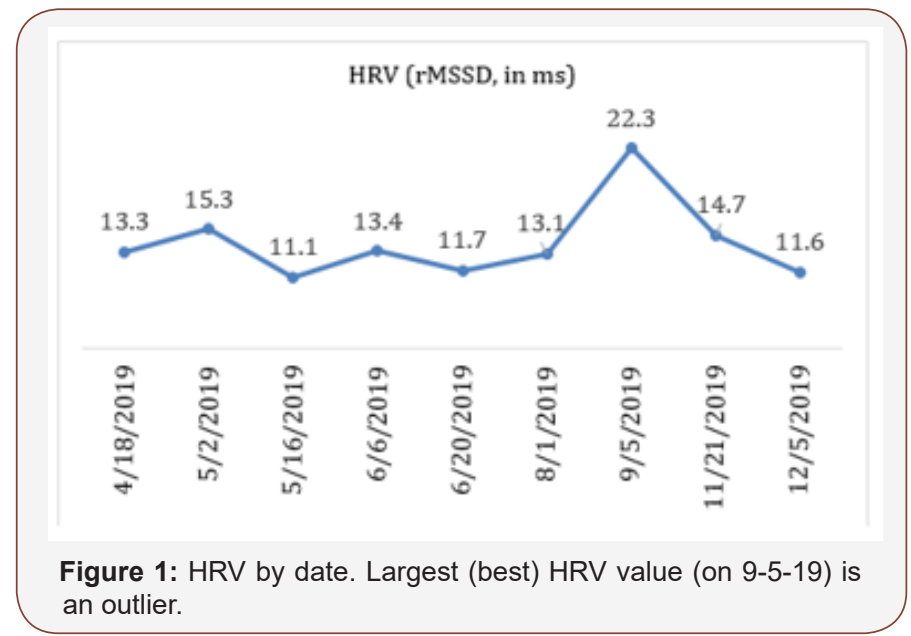

HRV was recorded using the Heart Rate Variability Logger app [5] in conjunction with the Kyto ear clip sensor [6]. The sensor sends a Bluetooth signal to the smartphone app and the set-up has good agreement with standard ECG technology [6-7]. A recording lasted 1 minute with the patient in the seated position after a seated rest period of at least 5 minutes. The time domain measure of root mean square of successive differences between heart beats 
(rMSSD), measured in milliseconds (ms) was used. The terms rMSSD and HRV are used interchangeably in this paper. For her age, a minimum healthy rMSSD value is approximately 20-28 ms [8-9]. By June though it became apparent that neurological progress was unsatisfactory (Figure 1).

The author recommended a second natural method of improving HRV, namely, exercise. The patient began running short distances at this time, namely, twice around her block where she lived. By the September check-up there was substantial improvement (increase) in the HRV to $22.3 \mathrm{~ms}$ (Figure 1).

Curious as to whether this improvement was statistically unusual, the author performed an outlier analysis using the interquartile method [10]. The method used the conventional factor of 1.5 for moderate outlier detection, multiplying it by the interquartile range (IQR) to obtain the fence limits:

Lower fence $=$ Quartile $1-(1.5 * I Q R)$

Upper fence $=$ Quartile $3+(1.5 * I Q R)$

Observed values outside the fences are considered outliers (statistically unusual findings).

The improved HRV reading on the 9-5-19 visit was calculated as an outlier, as it was greater than the upper fence in outlier analysis (Table 1).

Table 1: Summary statistics.

\begin{tabular}{|c|c|}
\hline Date & HRV (rMSSD, in ms) \\
\hline $4 / 18 / 2019$ & 13.3 \\
\hline $5 / 2 / 2019$ & 15.3 \\
\hline $5 / 16 / 2019$ & 11.1 \\
\hline $6 / 6 / 2019$ & 13.4 \\
\hline $6 / 20 / 2019$ & 11.7 \\
\hline $8 / 1 / 2019$ & 13.1 \\
\hline $9 / 5 / 2019$ & 22.3 \\
\hline $11 / 21 / 2019$ & 14.7 \\
\hline $12 / 5 / 2019$ & 11.6 \\
\hline Quartile 1 & 11.7 \\
\hline Quartile 3 & 14.7 \\
\hline IQR & 3 \\
\hline Lower fence & 7.2 \\
\hline Upper fence & 19.2 \\
\hline Smallest & 11.1 \\
\hline Largest & 22.3 \\
\hline
\end{tabular}

Following this, though, the HRV decreased (worsened) as it was before. Thus, the improvement was only temporary.

\section{Discussion}

Initially, HRV was consistently less-than-optimal HRV, from April to the August 1 visit (Figure 1). Perhaps another technique of spinal adjustment may have obtained better results during this time. As a medical doctor told me years ago though, "nothing works for everybody." The author's other published works using HRV are examples of his chiropractic success in other cases [11-13].
There was a hint on the September 5 visit that the exercise was working, that it was producing a beneficial autonomic effect evidenced by the HRV improvement. The improvement was substantiated by the outlier analysis. However, the HRV returned to the worsened state on the next visits (November 21 and December 5). Nonetheless, the improvement noted on September 5 is still a clue that the exercise was the right call and that it should be continued. The author plans to work with the patient to explore possible modifications to her exercise program (e.g., increase the distance and/or intensity of the running). The hope is to make the improvement long lasting.

\section{Acknowledgement}

The author thanks the patient for her consent in publishing her case.

\section{Conflict of Interest}

No Conflict of interest.

\section{References}

1. Zulfiqar U, Jurivich DA, Gao W, Singer DH (2010) Relation of high heart rate variability to healthy longevity. American Journal of Cardiology 105(8): 1181-1185.

2. Zhang J, Dean D, Nosco D, Strathopulos D, Floros M (2006) Effect of chiropractic care on heart rate variability and pain in a multisite clinical study. Journal of Manipulative and Physiological Therapeutics 29: 267 274.

3. Younes M, Nowakowski K, Didier-Laurent B, Gombert M, Cottin F (2017) Effect of spinal manipulative treatment on cardiovascular autonomic control in patients with acute low back pain. Chiropractic \& Manual Therapies 25: 33.

4. Stein PK, Ehsani AA, Domitrovich PP, Kleiger RE, Rottman JN (1999) Effect of exercise training on heart rate variability in healthy older adults. American Heart Journal 138(3 Pt 1): 567-576.

5. Altini M (2019) Heart rate variability Logger - app details. at: https:// www.marcoaltini.com/blog/heart-rate-variability-logger-app-details.

6. Vescio B, Salsone M, Gambardella A, Quattrone A (2018) Comparison between electrocardiographic and earlobe pulse photoplethysmographic detection for evaluating heart rate variability in healthy subjects in short- and long-term recordings. Sensors 18: 844.

7. Plews DJ, Scott B, Altini M, Wood M, Kilding AE, Laursen PB (2017) Comparison of heart rate variability recording with smart phone photoplethysmographic Polar H7 chest strap and electrocardiogram methods. International Journal of Sports Physiology 12(10): 1-17.

8. Kim GM, Woo JM (2011) Determinants for heart rate variability in a normal Korean population. Journal of Korean Medical Science 26: 12931298.

9. Hyun JB, Chul HC, Jaegeol C, Jong MW (2015) Reliability of ultra-shortterm analysis as a surrogate of standard 5-min analysis of heart rate variability. Telemedicine and e-health 21(5): 404-414.

10. Devore J, Peck R (2001) Statistics: The exploration of data. 4th Edition. Pacific Grove, CA: Duxbury.

11. Hart J (2019) Heart rate variability following spinal adjustment: A practice-based study. Internet Journal of Neurology 21(1).

12. Hart J (2019) Improvement in heart rate variability following spinal adjustment: A case study in statistical methodology for a single office visit. International Journal of Statistics in Medical Research 8: 17-22.

13. Hart J (2019) Inter-quartile analysis of resting heart rate and heart rate variability following spinal adjustment: A case study. Cardiovascular Systems. 